\title{
TIDAL INTERACTIONS IN M81 GROUP
}

\author{
M.S. YUN \\ National Radio Astronomy Observatory \\ P.O Box 0, Socorro, NM 87801 (USA)
}

\section{Introduction}

At a distance of $3.3 \mathrm{Mpc}, \mathrm{M} 81$ group is one of the nearest groups of galaxies and thus an ideal place to study various galactic phenomena. Observations at all wavelengths can be made with high sensitivity and excellent spatial resolution $\left(1^{\prime \prime}=15 \mathrm{pc}\right)$. The three core members M81, M82, and NGC 3077 are known to be closely interacting from the $21 \mathrm{~cm} \mathrm{HI}$ emission studies (van der Hulst 1979, Yun et al. 1993, 1994), and all three galaxies show evidence for an AGN or starburst activity, possibly induced by the on-going tidal interactions. A more distant member NGC 2976 also shows evidence for tidal interaction within the group (Appleton et al., 1981). Therefore M81 group is an excellent test ground for studying tidal interaction and tidally induced activities.

The central six main members of the group are listed in Table 1. Their optical appearances suggest little evidence for any on-going tidal interactions (see Fig. 1). The aperture synthesis maps of their $21 \mathrm{~cm} \mathrm{HI}$ emission,

TABLE 1. M81 Group Members

\begin{tabular}{lccccccc}
\hline name & $\begin{array}{c}\mathrm{RA} \\
(\mathrm{B} 1950)\end{array}$ & $\begin{array}{c}\text { Dec } \\
(\mathrm{B} 1950)\end{array}$ & $\begin{array}{c}v_{\text {hel }} \\
(\mathrm{km} / \mathrm{s})\end{array}$ & $\begin{array}{c}\log L_{\mathrm{B}} \\
\left(L_{\odot}\right)\end{array}$ & $\begin{array}{c}\log L_{\mathrm{FIR}} \\
\left(L_{\odot}\right)\end{array}$ & $\begin{array}{c}D_{25} \\
\left({ }^{\prime}\right)\end{array}$ & $\begin{array}{c}d_{\mathrm{p}}(\mathrm{M} 81) \\
(\mathrm{kpc})\end{array}$ \\
\hline M81 & $09: 51: 27.3$ & $69: 18: 08$ & -34 & 10.28 & 8.33 & 27 & - \\
M82 & $09: 51: 43.5$ & $69: 55: 01$ & +203 & 9.69 & 10.24 & 11 & 33 \\
NGC 3077 & $09: 59: 19.9$ & $68: 58: 30$ & +14 & 9.05 & 8.43 & 5.4 & 42 \\
NGC 2976 & $09: 43: 11.5$ & $68: 08: 45$ & +3 & 9.11 & 8.40 & 5.9 & 74 \\
HO I & $09: 36: 00.9$ & $71: 24: 55$ & +136 & 8.03 & - & 3.6 & 135 \\
IC 2574 & $10: 24: 41.3$ & $68: 40: 18$ & +47 & 9.10 & 7.86 & 13 & 164 \\
\hline
\end{tabular}




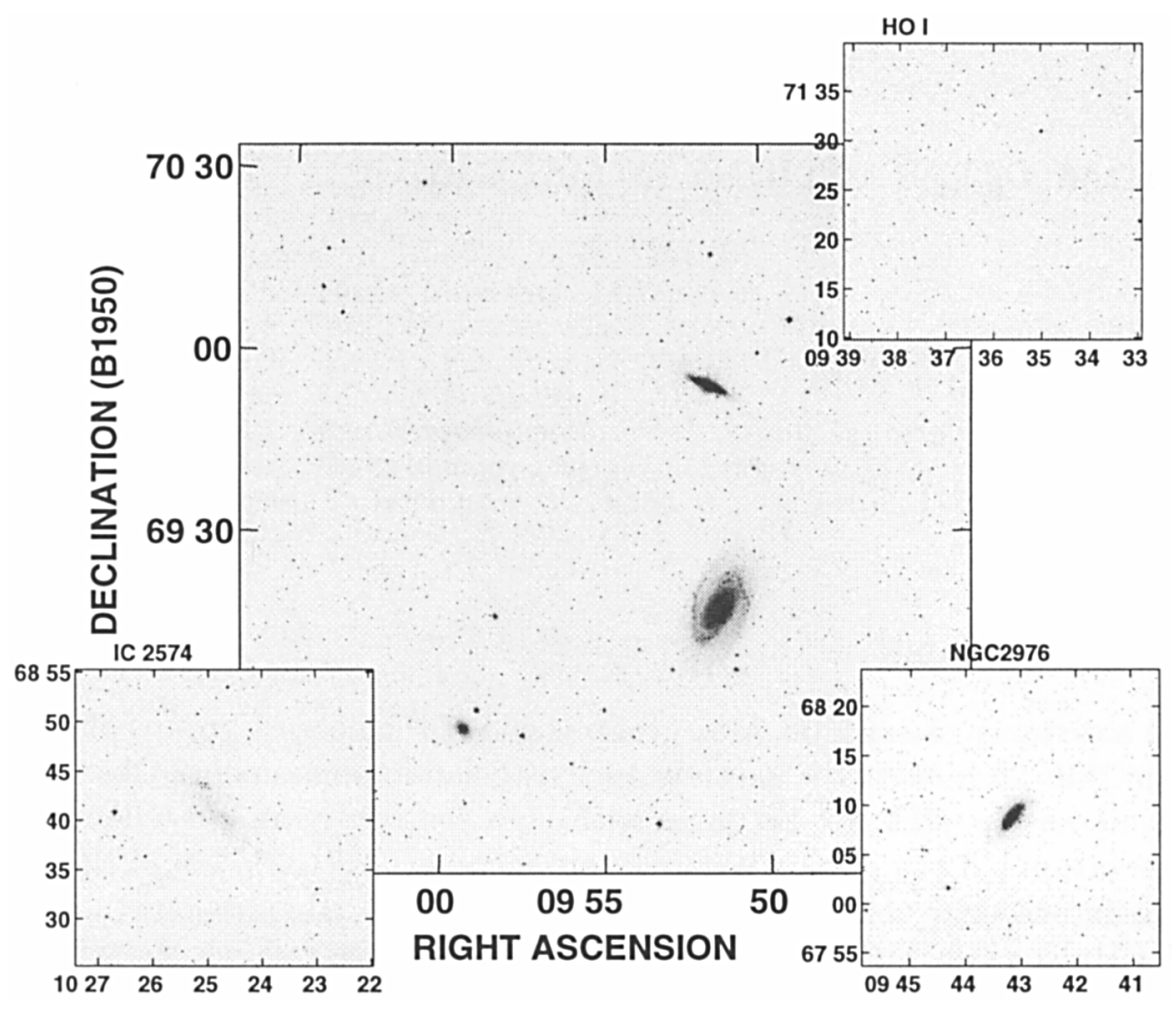

Figure 1. Optical images of the M81 Group galaxies.

however, show clear evidence for on-going tidal disruptions in M81, M82, NGC 3077, and NGC 2976 (see Fig. 2 and Appleton et al. 1981). The HI distribution in the more distant members HO I and IC 2574 appears undisturbed (D. Westpfahl, private communication). It is somewhat peculiar that the most luminous member M81 also has the most blueshifted velocity within the group (Table 1), but this probably reflects its orbital motion with respect to the two nearby companions M82 and NGC 3077. The mean group line-of-sight velocity, $55 \mathrm{~km} \mathrm{~s}^{-1}$, is nearly identical to the mean of the three outer members, $62 \mathrm{~km} \mathrm{~s}^{-1}$.

In the remainder of this paper, I will focus only on the tidal interactions involving the inner most three members (M81, M82, \& NGC 3077) because the HI data suggest that their interactions are the most current. The HI data also suggest that NGC 2976 has passed through the group center in the recent past, but the tidal remnants associated with this older interaction are likely largely erased by the more recent ones. First, the $21 \mathrm{~cm} \mathrm{HI}$ observations of the three galaxies are summarized in $\S 2$. Numerical model- 
TABLE 2. Summary of the HI Observations

\begin{tabular}{lccccc}
\hline name & $\begin{array}{c}N_{\mathrm{HI}, \text { peak }} \\
\left(10^{21} \mathrm{~cm}^{-2}\right)\end{array}$ & $\begin{array}{c}M_{\mathrm{HI}}\left(10^{9} M_{\odot}\right) \\
\mathrm{VLA}\end{array}$ & $\mathrm{ADS}^{\dagger}$ & $\begin{array}{c}\left\langle V_{\mathrm{HI}}\right\rangle \\
(\mathrm{km} / \mathrm{s})\end{array}$ & $\begin{array}{c}\Delta V \\
(\mathrm{~km} / \mathrm{s})\end{array}$ \\
\hline M81 & $10.6 \pm 0.2$ & $2.81 \pm 0.56$ & $2.19 \pm 0.22$ & -35 & $30 \sim 45$ \\
M82 & $10.3 \pm 0.2$ & $0.80 \pm 0.16$ & $0.72 \pm 0.07$ & +230 & $40 \sim 140$ \\
NGC 3077 & $10.7 \pm 0.2$ & $0.69 \pm 0.14$ & $1.00 \pm 0.10$ & +0 & $30 \sim 40$ \\
Concentration I & $7.8 \pm 0.2$ & $0.31 \pm 0.06$ & $0.20 \pm 0.05$ & $+50 \sim+150$ & $45 \sim 100$ \\
Concentration II & $5.1 \pm 0.2$ & $0.26 \pm 0.05$ & - & +120 & $25 \sim 30$ \\
S. Tidal Bridge & $2.4 \pm 0.2$ & $0.26 \pm 0.05$ & - & $-200 \sim+0$ & $10 \sim 30$ \\
N. Tidal Bridge & $1.6 \pm 0.2$ & $0.20 \pm 0.04$ & - & $+0 \sim+120$ & $20 \sim 30$ \\
\hline
\end{tabular}

† Single dish measurements by Appleton, Davies, \& Stephenson (1981).

ing of the tidal interactions and its implications are discussed in $\S 3$ and $\S 4$. Finally, the summary is given in $\S 5$.

\section{HI Observations}

The $21 \mathrm{~cm} \mathrm{HI} \mathrm{emission} \mathrm{map} \mathrm{of} \mathrm{the} \mathrm{central} 2^{\circ}$ region of the M81 group at $1^{\prime}$ resolution (Fig. 2) is produced using a mosaic of 12 separate pointings at the Very Large Array ${ }^{1}$ (VLA; see Yun 1992 and Yun et al. 1994 for more detailed description of the observations). A total of $5.6 \times 10^{9} M_{\odot}$ of atomic hydrogen is detected in this group, mostly concentrated on the three individual galaxies. The two bright HI knots found east of M81 disk ("Concentrations I \& II") have the HI masses comparable to a dwarf galaxy, and the Concentration I may be associated with HO IX, a clump of stars previously suggested to be a satellite companion of M81. The gas kinematics are consistent with simple disk rotations or tidal perturbations superposed on a disk rotation in the three galaxies. The derived properties of the individual HI features are summarized in Table 2.

By far the most remarkable features seen in $\mathrm{HI}$ are the tidal bridges connecting all three galaxies. The bridge connecting M81 and NGC 3077 ("South Tidal Bridge") was previously mapped by van der Hulst (1979), and this feature appears to loop around NGC 3077 and pointing toward M82 ("North Tidal Bridge"). Both of these tidal features are massive - each $2 \times 10^{8} M_{\odot}$ in $\mathrm{HI}$ mass and $\geq 30 \mathrm{kpc}$ in length. The HI complex associated with NGC 3077 is displaced by $4 \mathrm{kpc}$ to the southeast of the stellar galaxy, and a bright loop of $\mathrm{HI}$ connects the HI peak with the stellar peak. The HI

\footnotetext{
${ }^{1}$ The National Radio Astronomy Observatory is a facility of the National Science Foundation operated under cooperative agreement by Associated Universities, Inc.
} 

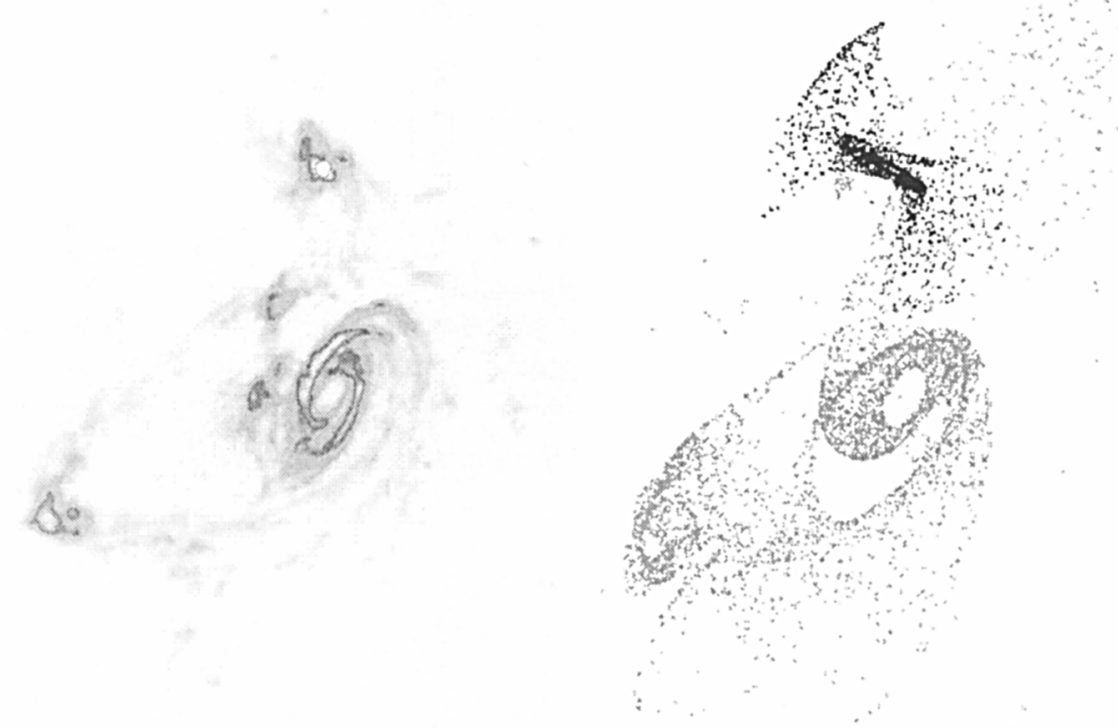

Figure 2. Comparison of the $21 \mathrm{~cm} \mathrm{HI}$ emission (left) and the numerical simulation (left).

disk of M82 is severely disrupted, contributing to the HI bridge connecting with M81 (see Yun et al. 1993 for a detailed study). These tidal features are completely absent in optical light (Fig. 1), and one would not have guessed that these three galaxies are strongly interacting based on the optical image alone. This in turn suggests that the frequency of tidal interactions among galaxies may far exceed any estimate based on optical images alone.

\section{Numerical Modeling}

Disk galaxies are highly coherent objects in phase space, and therefore numerical simulation of tidally interacting disk galaxies can be a relatively straightforward task as long as gravity is the dominant force, even in cases where tidal features display complex morphology and kinematics. The pioneering work by Toomre \& Toomre (1972) has convincingly demonstrated that the filamentary features associated with close pairs of galaxies are indeed the tidal debris resulting from galaxy-galaxy collisions. They have further suggested that such tidal disruptions may "stoke the furnace" and result in enhanced starburst or AGN activities. Recent reviews on numerical modeling of galaxy interaction and mergers are found in Barnes (1998) and also in the contributions by J. Barnes and C. Mihos in this volume.

Modeling an interacting pair of galaxies is an extension of a simple 
TABLE 3. Input parameters for the modeling.

\begin{tabular}{lccc}
\hline & M81 & M82 & NGC 3077 \\
\hline RA (1950) & $09^{h} 51^{m} 30^{s}$ & $09^{h} 51^{m} 44^{s}$ & $09^{h} 59^{m} 22^{s}$ \\
Dec. (1950) & $+69^{\circ} 18^{\prime} .3$ & $+69^{\circ} 54^{\prime} .9$ & $+68^{\circ} 58^{\prime} .5$ \\
Relative Velocity $\left(\mathrm{km} \mathrm{s}^{-1}\right)$ & 0 & +230 & +36 \\
Total Mass $\left(10^{10} M_{\odot}\right)$ & 20 & $1-4$ & $1-4$ \\
Disk Radius $(\mathrm{kpc})$ & 36 & 17 & 17 \\
Disk Inclination & $59^{\circ}$ & $82^{\circ}$ & $45^{\circ}$ \\
Disk P.A. & $150^{\circ}$ & $28^{\circ}$ & $225^{\circ}$ \\
$\alpha^{\dagger}(\mathrm{kpc})$ & 0.3 & 0.1 & 0.1 \\
\hline
\end{tabular}

${ }^{\dagger}$ softening parameter in Plummer potential.

2-body interaction, which is a problem with a well known solution. The modeling a system of three interacting galaxies is a far more difficult problem since gravitational interaction involving three bodies has no simple analytical solution. Therefore, the problem of modeling the tidal interactions among M81, M82, and NGC 3077 is first approached as a problem of understanding two separate pair-wise interactions. This may be a reasonable approximation since M81 is much more massive than its two companions. The modeling is further simplified by performing only the "restricted 3-body" calculation (tracing the motions of test particles within a time dependent potential, as in Toomre \& Toomre 1972) rather than attempting full N-body calculations with self-consistent galaxy models (e.g. Barnes \& Hernquist 1996). Some of the consequences of using these simplifications are discussed below.

The code, originally developed by N. Brouillet (L'université Bordeaux), computes the motions of a disk of massless test particles that are initially in circular orbit about its central (Plummer) potential. The initial parameter searches roughly determine the M81-M82 and M81-NGC3077 orbits separately, and then a full three galaxy interaction and the resulting responses of the disk particles are realized by combining the pair-wise solutions. The input parameters for the model calculations are listed in Table 3, and the best model solution obtained is shown in comparison with the HI data in Fig. 2. The numerical simulation not only matches the observed HI distribution well, but it also matches the velocity field also (not shown here) even though no effort was made to do so. The best model parameters are listed in Table 4.

The details of the tidal interactions are shown in Fig. 3 by displaying the particles representing three galaxies separately along with the traces 
TABLE 4. Best 3-body numerical model parameters.

\begin{tabular}{lccc}
\hline & M81 & M82 & NGC 3077 \\
\hline Mass $\left(10^{10} M_{\odot}\right)$ & 20 & 4 & 2 \\
Orbit Eccentricity & - & 1.5 & 0.7 \\
Perigal. Distance $(\mathrm{kpc})$ & - & 25 & 16 \\
Time since pericenter & - & $2.2 \times 10^{8} \mathrm{yrs}$ & $2.8 \times 10^{8} \mathrm{yrs}$ \\
Orbit Inclinations, $i$ & - & $10^{\circ}$ & $135^{\circ}$ \\
Perigal. Argument, $\omega$ & - & $235^{\circ}$ & $270^{\circ}$ \\
Orbit Longitude, $\lambda$ & - & $265^{\circ}$ & $175^{\circ}$ \\
\hline
\end{tabular}

of the companion orbits. The tidal disruption of the M82 disk is done entirely by M81 alone while the disruption of both M81 and NGC 3077 disks require both of their two companions. The key difference between this simulation from the previous simulation of M81 group (Cottrell 1977, van der Hulst 1977, Killian 1978, Brouillet et al. 1991) is the inclusion of an HI disk surrounding NGC 3077. All attempts to produce the North Tidal Bridge (between M82 and NGC 3077) as the tidally disrupted outer disk of M81 failed, and NGC 3077 was given its own disk in order to reproduce this feature (nearly $10^{9} M_{\odot}$ of $\mathrm{HI}$ is associated with NGC 3077, further justifying that NGC 3077 initially possessed its own disk, which is now nearly unrecognizable). The original HI disk of NGC 3077 is first disrupted by M81 and is then stretched out further like a streamer during the subsequent passage by M82 (see a GIF movie of this interaction at http://www.aoc.nrao.edu/ myun/movie.gif). The apparent smooth connection of the North Tidal Bridge (NTB) with the South Tidal Bridge (STB - tidally disrupted outer disk of M81) near NGC 3077 seems fortuitous, but it is not at all an accident since tidal tails tend to point back and finger their perturbers (M82 for the NTB and NGC 3077 for the STB).

\section{Discussions}

The most interesting physical parameters derived from this simulation are the time scales of interactions. The time since the nearest approach for M82 and NGC 3077 are $2.2 \times 10^{8}$ and $2.8 \times 10^{8}$ years, respectively. These agree very well with the estimated ages of on-going starbursts in M82 and NGC 3077 (see Rieke et al. 1980), and such a good agreement is a strong support for the idea that the tidal disruptions are indeed responsible for the "stoking of the furnaces". The actual inflow of the gas into the nuclear region is likely driven by a hydrodynamic process not included in the present 

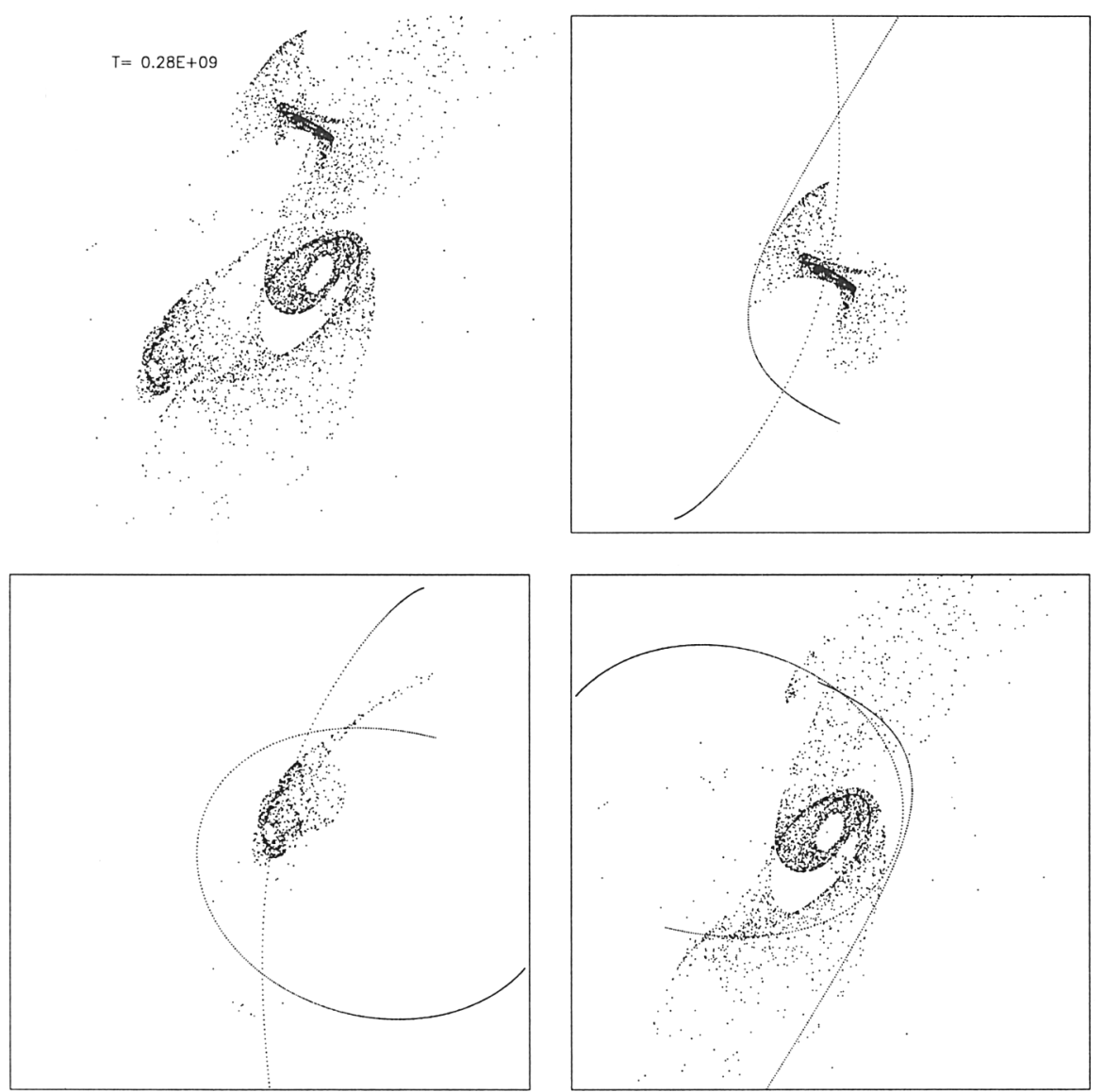

Figure 3. Tidal disruptions of the individual galaxies are shown separately to illustrate the course and nature of tidal interactions and the resulting tidal features.

model, but such gas inflow has been seen in other simulations such as by Mihos \& Hernquist (1996) and Barnes \& Hernquist (1996).

The details of the inner disk of M81 (and the others) are not well reproduced by this simulation, but this is the result of using a Plummer potential rather than a self-consistent galaxy model with full N-body calculation. If self-gravity and hydrodynamical effects are included in describing the response of the HI disks, the tidal features reproduced by the simulation should have sharper appearances as seen in the HI map. The full N-body calculations using self-consistent model galaxies, on the other hand, have demonstrated that dynamical dissipation of orbital energy and angular momentum can significantly alter the course of tidal interaction, often leading to mergers of galaxies (see Barnes 1998 and references therein). If the dissi- 
pation of orbital energy and angular momentum also occurs in this system, then the parabolic orbits describing the observed interactions are indeed only the approximations of the motions of individual galaxies past their initial collisions. A full N-body simulation of this system has been attempted previously using the orbits similar to derived here, but this simulation resulted in the mergers of the companions onto M81, probably because the dynamical dissipation associated with the truncated isothermal model halo used was too efficient (Yun 1992). The evolution of outer tidal features and dynamical decay of the orbits depend critically on the physical nature of the halo, and I speculate that simulating non-merging interacting systems using self-consistent $\mathrm{N}$-body model will first require a better understanding of the nature of the galactic halos.

\section{Summary}

A tidal interaction scenario involving M81, M82, and NGC 3077 was derived using a restricted 3-body calculation. This scenario can successfully account for the complex system of tidal features seen in the $21 \mathrm{~cm}$ HI emission map, despite the multitude of simplifying assumptions incorporated into the modeling. The derived time scale for tidal interactions agree very well with the estimated ages of starbursts seen in M82 and NGC 3077, lending further support that these starburst are the direct results of the tidal disruptions suffered within last $3 \times 10^{8}$ years.

\section{References}

Appleton, P.N., Davies, R.D., Stephenson, R.J. 1981, MNRAS, 195, 327

Barnes, J.E. 1988, ApJ, 331, 669

Barnes, J.E. 1998, in Galaxies: Interactions and Induced Star Formation, eds. D. Friedli, L. Martinet, \& D. Pfenninger (Springer-Verlag)

Barnes, J.E., Hernquist, L. 1996, ApJ, 471, 115

Brouillet et al. 1991, A\&A, 242, 35

Cottrell, G.A. 1977, MNRAS, 178, 577

Killian, D.J. 1978, PhD thesis, University of Florida

Mihos, J.C., Hernquist, L. 1996, ApJ, 464, 641

Rieke et al. 1980, ApJ, 238,24

Toomre, A. \& Toomre, J. 1972, ApJ, 178, 623

van der Hulst, J.M. 1977, PhD thesis, University of Groningen

van der Hulst, J.M. 1979, A\&A, 75, 97

Yun, M.S. 1992, PhD thesis, Harvard University

Yun, M.S., Ho., P.T.P., Lo, K.Y. 1993, ApJ, 411, L17

Yun, M.S., Ho., P.T.P., Lo, K.Y. 1994, Nature, 372,530 